\title{
MODELACIÓN DE LA PARTICIÓN DE LLUVIA EN ESPECIES ARBÓREAS Y ARBUSTIVAS DE CLIMA MEDITERRÁNEO
}

\author{
Pérez Arellano, R. $^{1}$, Serrano Gómez A. ${ }^{2}$, Roldan Cañas, $J^{3}{ }^{3}$, Moreno Pérez, M.F. ${ }^{4}$ \\ ${ }^{1}$ Doctorando, Universidad de Córdoba, Dpto. Agronomía (Área Ingeniería Hidráulica), Edif. \\ Leonardo Da Vinci (Campus Rabanales), 14071 Córdoba, g82pearr@uco.es \\ 2 Ingeniero Agrónomo. Dpto. Agronomía (Área Ingeniería Hidráulica), Edif. Leonardo Da \\ Vinci (Campus Rabanales), 14071 Córdoba, g82segoa@uco.es \\ ${ }^{3}$ Catedrático, Universidad de Córdoba, Dpto. Agronomía (Área Ingeniería Hidráulica), Edif. \\ Leonardo Da Vinci (Campus Rabanales), 14071 Córdoba, jroldan@uco.es \\ ${ }^{4}$ Profesora Contratada Doctora, Universidad de Córdoba, Dpto. Agronomía (Área Ingeniería \\ Hidráulica), Edif. Leonardo Da Vinci (Campus Rabanales), 14071 Córdoba, mfatima@uco.es
}

\section{Resumen}

El estudio de la intercepción de la lluvia por el dosel de la vegetación es de gran importancia en el balance hídrico de las cuencas. Gran parte de la precipitación incidente vuelve a la atmósfera en forma de evaporación, esto implica que sólo un porcentaje de la cantidad de lluvia que cae durante un evento formará parte del caudal de la cuenca, ya sea como caudal superficial o subterráneo. El objetivo de este trabajo ha sido el estudio de los modelos de interceptación de Rutter y Gash y su aplicación a dos especies predominante en la vegetación de la cuenca de "El Cabril", en el sur de España, como son el matorral, compuesto en su mayoría de jaras (Cistus ladanifer) y el pino (Pinus pinea). Se midió la precipitación incidente, la trascolación y la escorrentía cortical en ambas especies durante cuatro años (entre octubre de 2010 y junio de 2014). Los resultados muestran que la pérdida de interceptación es mayor en el pino (29.6\% de la precipitación incidente) que en la jara ( $17 \%$ de la precipitación incidente). El modelo analítico de Rutter modificado por Valente ofreció los mejores resultados, siendo las diferencias entre las medidas observadas y las simuladas inferiores al $2 \%$.

\section{Abstract}

The study of rainfall interception by the vegetation canopy is of great importance in the water balance of watersheds, because much of the precipitation returns to the atmosphere as evaporation. This implies that only a percentage of the amount of rain precipitated during an event will be part of the flow of the basin, either as surface or groundwater flows. The aim of this work has been to study the Rutter and Gash interception models and to apply them to the predominant vegetation in the watershed of "The Cabril", Southern Spain, including: scrub, composed mostly of rockrose (Cistus ladanifer), and arboreal masses of pine trees (Pinus pinea). Gross rainfall, throughfall and stemflow were measured in these species over four years (from October 2010 to June 2014). The results show that the interception loss is higher in the pine stand (27\% of gross rainfall) than in the cistus stand (17\% of gross rainfall). The Rutter analytical model modified by Valente gave the best results, since the differences between the observed measures and the simulated measures were lower $2 \%$. 


\section{1- Introducción}

La vegetación juega un importante papel en la hidrología de una cuenca, ya que afecta al balance de agua de la superficie en todos sus componentes: interceptación de lluvia incidente, infiltración, escorrentía superficial y evapotranspiración. Predecir el efecto de la vegetación sobre los recursos hídricos requiere de mediciones y modelos para extrapolar los resultados de esas mediciones en el tiempo y en el espacio.

El primer intento de modelar las pérdidas por interceptación fue realizado por Horton (1919), tanto para bosques naturales como para matorrales y arbustos. Horton observó que al inicio de los eventos de lluvia, el porcentaje de precipitación que alcanza el suelo es pequeño, aumentando conforme avanza la duración de la tormenta. En su trabajo estudió también la importancia de la forma de la hoja y el ángulo que forman respecto a la horizontal, y como cuando una vez se alcanza la máxima capacidad de almacenamiento en la hoja, el agua es drenada hacia las partes bajas.

Décadas más tarde, el estudio de la interceptación realizado por Rutter (Rutter et al., 1971) hizo posible el desarrollo de modelos basados en la física de la interceptación de lluvia. A partir de Rutter se derivan una serie de modelos como son el modelo analítico de Gash (1979), los modelos numéricos de Mulder (1985) y Whitehead y Kelliher (1991), y en menor medida el modelo estocástico de Calder (1986).

Para la elaboración de estos modelos es necesario disponer de datos como la evaporación, el coeficiente de trascolación, el porcentaje de interceptación por ramas y troncos, la resistencia aerodinámica y la capacidad de almacenamiento del dosel (Rutter et al., 1975). El modelo propuesto por Gash (1979) conserva algo de la simplicidad de un enfoque empírico, y también gran parte del razonamiento físico adoptado en el modelo numérico de Rutter (Zhang et al., 2006). Además simplifica el proceso de cálculo de la obtención de la interceptación, pasando de mediciones horarias a mediciones diarias.

Estos modelos se han aplicado en una amplia gama de tipos de bosques para estudiar su aplicación a la hora de estimar la interceptación. Por ejemplo, se han realizado estudios en coníferas (Murakami, 2007), en árboles caducifolios (Gerrits et al., 2010), en bosques subtropicales (Zhang et al. 2006) y tropicales (Wallace y McJannet, 2006). En clima mediterráneo se han aplicado en bosque de encino (Quercus ilex) (Pereira et al. 2009), y se han adaptado a bosques dispersos de eucaliptos y pinos (Valente et al., 1997), y de robles (Muzylo et al., 2012)

En este trabajo se seleccionaron los modelos clásicos de Rutter y de Gash, para realizar la simulación de la interceptación y comparar así un modelo numérico frente a otro analítico. Además se ha estudiado la aplicabilidad de la versión adaptada por Valente de estos modelos a bosques dispersos. Para analizar la universalidad de estos modelos, se han tomado los datos de una especie arbórea (Pinus pinea) y otra arbustiva (Cistus ladanifer) en una cuenca Mediterránea situada en el municipio de Hornachuelos (Córdoba).

En este trabajo se han hecho algunas modificaciones a los modelos para adaptarlos a la vegetación utilizada y las características climáticas de la zona (Serrano, 2013), consistentes en establecer límites a la evaporación desde el tronco y la evaporación desde el dosel en ambas versiones. En el caso del modelo original, también se ha considerado los límites establecidos en el drenaje de la cubierta, mejora que ya había sido propuesta en un trabajo anterior de Gerrits (2010). 


\section{2- Materiales y Métodos}

Para la aplicación de los modelos de interceptación son necesarios datos horarios de parámetros climáticos tales como la velocidad del viento, la radiación solar, la temperatura y la humedad relativa, además de las medidas de campo de los diversos componentes del balance de agua. Los primeros han sido tomados de una estación agroclimática instalada en el "El Cabril" (Hornachuelos, Córdoba), mientras que los componentes del balance de agua (precipitación incidente, trascolación, escorrentía cortical e interceptación) se midieron en parcelas experimentales de Pinus pinea y Cistus ladanifer para los eventos de lluvia que tuvieron lugar entre octubre de 2010 y junio de 2014 (Moreno-Pérez et al.; 2014).

El modelo de Rutter formula un balance hídrico del dosel y el tronco, en donde las entradas son la precipitación incidente a cada hora y los parámetros meteorológicos necesarios para estimar la evaporación potencial (Fig. 1). La estructura de la cubierta queda descrita por el coeficiente de escurrimiento libre $(p)$, el coeficiente de partición de agua del escurrimiento fustal $\left(p_{t}\right)$, la capacidad de almacenamiento de la cubierta $(S)$ y la capacidad de almacenamiento de los troncos $\left(S_{t}\right)$. 


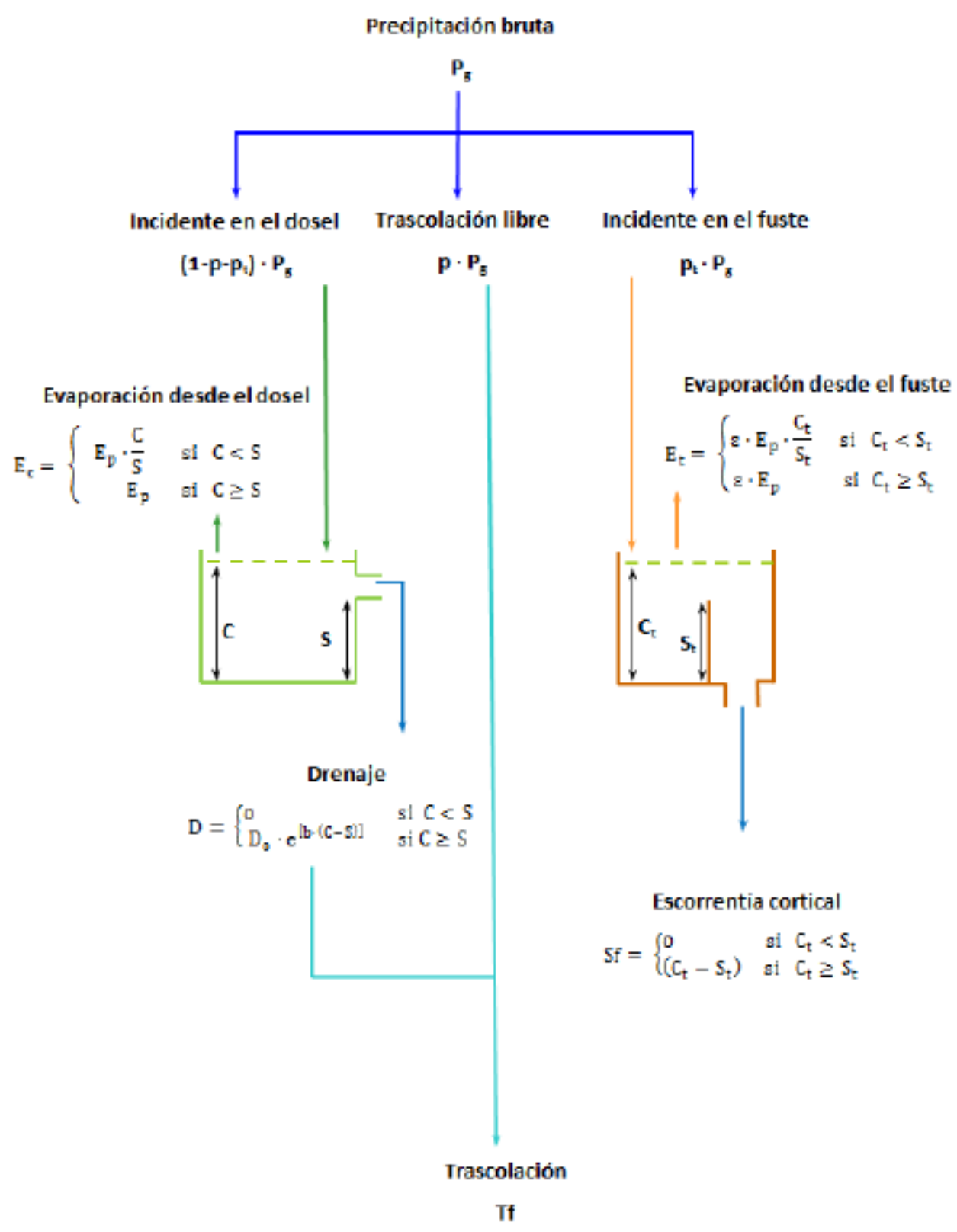

Figura 1. Modelo de Rutter esquematizado

La trascolación, el escurrimiento fustal y las pérdidas por intercepción se obtienen de un balance de agua en el dosel y en el fuste, a partir de las siguientes ecuaciones 1 y 2 :

$$
\begin{aligned}
& (1-p-t) \int R d t=\int D d t+\int E_{c} d t+\Delta C \\
& p t \int R d t=S f+\int E_{t}+\Delta C t
\end{aligned}
$$

, donde $R(\mathrm{~mm})$ es la intensidad de precipitación, $D(\mathrm{~mm} / \mathrm{h})$ es la tasa de drenaje del dosel, $E_{c}(\mathrm{~mm} / \mathrm{h})$ es la tasa de evaporación de la lluvia interceptada por el dosel, $\Delta C(\mathrm{~mm})$ es la variación en el almacenamiento del dosel, $S_{f}(\mathrm{~mm})$ es la escorrentía cortical, $E_{t}(\mathrm{~mm} / \mathrm{h})$ es la tasa de evaporación del agua interceptada por los troncos y $\Delta C_{t}(\mathrm{~mm})$ es la variación en el almacenamiento de los troncos.

La precipitación que incide en el dosel $(C)$ es calculada a partir de la diferencia entre la precipitación incidente $\left(P_{g}\right)$ y los componentes ya descritos $\left(1-p-p_{t}\right) \cdot P_{g}$. Cuando la cantidad 
de lluvia que incide en el dosel supera la capacidad de almacenamiento del mismo $(S)$ se produce el drenaje del dosel el cual, junto con la trascolación libre, constituye la trascolación total. Al igual que en el caso del dosel, cuando la cantidad de agua que incide en el fuste $\left(C_{t}\right)$ supera a la capacidad de almacenamiento del mismo $\left(S_{t}\right)$, se produce un nuevo flujo denominado escorrentía cortical $\left(S_{f}\right)$.

La tasa de drenaje del dosel queda descrita en la bibliografía como una función exponencial (Lloyd et al., 1988), y es calculada a partir de la ecuación 3:

$$
\begin{aligned}
& D=\left\{D_{0} \cdot \exp [b \cdot(C-S)] \text { si } C \geq S\right. \\
& D=0 \text { si } C<S
\end{aligned}
$$

, donde $D_{0}$ es la tasa de drenaje cuando $C=S$ y $b$ es un coeficiente empírico. Los valores de $D_{0}$ y $b$ utilizados en este estudio fueron tomados de por Rutter et al. (1971)

La cantidad de lluvia que queda retenida en el dosel y en el fuste durante un evento de lluvia, y es posteriormente evaporada, constituye lo que se denomina pérdidas por interceptación. Para el cálculo de estas pérdidas es fundamental determinar la evaporación potencial $\left(E_{p}\right)$, que se calcula utilizando la ecuación de Penman-Monteith (Monteith, 1965). Cuando la cantidad de lluvia que incide en el dosel $(C)$ es menor que la capacidad de almacenamiento del dosel $(S)$, la evaporación se reduce en proporción a $C / S$.

El modelo original de Gash (1979) es una simplificación del modelo de Rutter et al. (1971), basado en eventos singulares de lluvia, que se calibra utilizando datos meteorológicos horarios y se aplica luego con valores de lluvia diarios.

Este modelo considera que las precipitaciones ocurren como una serie de eventos de lluvia discretos, separados entre sí por un tiempo suficientemente largo como para que la planta quede completamente seca. Cada evento consta de tres fases distintas: una fase humectante, desde el inicio de la precipitación hasta que el dosel está saturado; una fase de saturación y una fase final de secado, que dura desde el final de la precipitación hasta que el dosel y los troncos estén completamente secos.

Las condiciones meteorológicas predominantes durante las dos primeras fases se supone que son los mismos y el promedio de los valores de intensidad de lluvia $(\bar{R})$ y la tasa de evaporación $(\bar{E})$ para las condiciones de dosel saturado se calculan para todo el período de simulación y después se aplican de forma generalizada a todos los eventos de lluvia individuales. $\bar{E}$ se calculó utilizando la ecuación de Penman-Monteith, como en el modelo Rutter. El modelo se aplicó a partir de datos horarios, suponiendo la ocurrencia de un único evento de lluvia por día. Los componentes del proceso de intercepción se calcularon como se indica en la Tabla 1.

Tabla 1.- Componentes de la interceptación en la versión original del modelo de Gash. 


\begin{tabular}{|c|c|c|}
\hline \multicolumn{3}{|l|}{ Pérdidas por interceptación desde el dosel: } \\
\hline $\begin{array}{l}\text { Para } m \text {, eventos insuficientes para saturar el } \\
\text { dosel }\left(P_{E} \leq P_{E^{\prime}}\right)\end{array}$ & & $\left(1-p-p_{t}\right) \sum_{j=1}^{m} P_{g, j}$ \\
\hline $\begin{array}{l}\text { Para } n \text {, eventos suficientes para saturar el } \\
\text { dosel }\left(P_{E}>P_{E}^{\prime}\right) \text { : }\end{array}$ & l) & $n\left\{\left(1-p-p_{t}\right) P_{g}^{\prime}-s\right\}$ \\
\hline I) Durante el humedecimiento del dosel & II) & $\frac{\varepsilon}{\bar{R}} \sum_{j=1}^{n}\left(P_{g, j}-P_{g}^{\prime}\right)$ \\
\hline $\begin{array}{l}\text { II) Una vez saturado el dosel } \\
\text { III) Evaporación una vez cesada la lluvia }\end{array}$ & III) & $n S$ \\
\hline \multicolumn{3}{|l|}{ Pérdidas por interceptación desde el fuste: } \\
\hline $\begin{array}{l}\text { Para } \mathrm{q} \text {, tormentas que saturan el fuste } \\
\left(\mathrm{P}_{\mathrm{g}} \geq \mathrm{P}_{\mathrm{g}}^{\prime \prime}\right)\end{array}$ & & $q S_{t}$ \\
\hline $\begin{array}{l}\text { Para } m+n-q \text {, tormentas que no saturan el } \\
\text { fuste }\left(\mathrm{P}_{\mathrm{g}}<\mathrm{P}_{\mathrm{g}}^{\prime \prime}\right)\end{array}$ & & $p_{t} \sum_{j=1}^{m+n-q} P_{g, j}$ \\
\hline
\end{tabular}

Los modelos originales de Rutter y de Gash tenderán, en teoría, a sobreestimar la pérdida por intercepción en los bosques con vegetación dispersa, ya que asumen que el área de evaporación se extiende a toda el área de estudio, mientras que el área de evaporación real es mucho más reducida en este tipo de cuencas forestales. Valente et al. (1997) hicieron una pequeña modificación de los modelos originales de Rutter y de Gash, asumiendo que la evaporación es nula en las áreas sin vegetación y que la evaporación desde el dosel se relaciona con la evaporación desde el fuste, cuando el dosel está saturado, siendo entonces la evaporación desde el dosel $(1-\varepsilon) E$, y la evaporación desde el fuste, $\varepsilon \cdot E$. Donde $\varepsilon$, normalmente vale 0,02 (Serrano, 2013).

El modelo de Rutter después de las modificaciones introducidas por Valente et al. (1997) para adaptarlo a masas forestales poco densas, aparece esquematizado en la figura 2. La estructura básica del nuevo modelo es similar al modelo original Rutter, sin embargo, los cálculos se realizan de forma independiente para cada una de las sub-áreas y no, como en la versión original, para toda el área de estudio. Además, considera que solamente incidirá agua en el fuste una vez se haya saturado el dosel, desviándose el exceso de agua hacia el fuste o cayendo al suelo mediante el drenaje, para lo cual aparece un nuevo parámetro, $p_{d}$, que se corresponde con la proporción de lluvia que se desvía hacia el tronco, y se sustituye la ecuación exponencial que describía el drenaje en la versión original por una ecuación lineal. La capacidad de almacenamiento $S$ y $S_{t}$ originales tienen que ser redefinidos como $S_{c}$ y $S_{t c}$, a partir de las ecuaciones 4 y 5 :

$$
\begin{aligned}
& S_{c}=S c \\
& S t c=S t c
\end{aligned}
$$

, donde $c$ representa la fracción de cobertura del dosel.

El modelo analítico de Gash revisado por Valente incluye modificaciones muy similares a los utilizados para el modelo de vegetación dispersa de Rutter. También se consideran dos sub-áreas distintas (área abierta y área cubierta), cada uno con la misma entrada de lluvia. La evaporación del dosel saturado se calcula, como en la versión original 
del modelo, a través de la ecuación de Penman-Monteith, aunque $\bar{E}$ es ahora sustituida por la expresión $(1-\varepsilon) \overline{E_{c}}$, y la evaporación de la zona abierta se supone que es cero (Tabla 2).

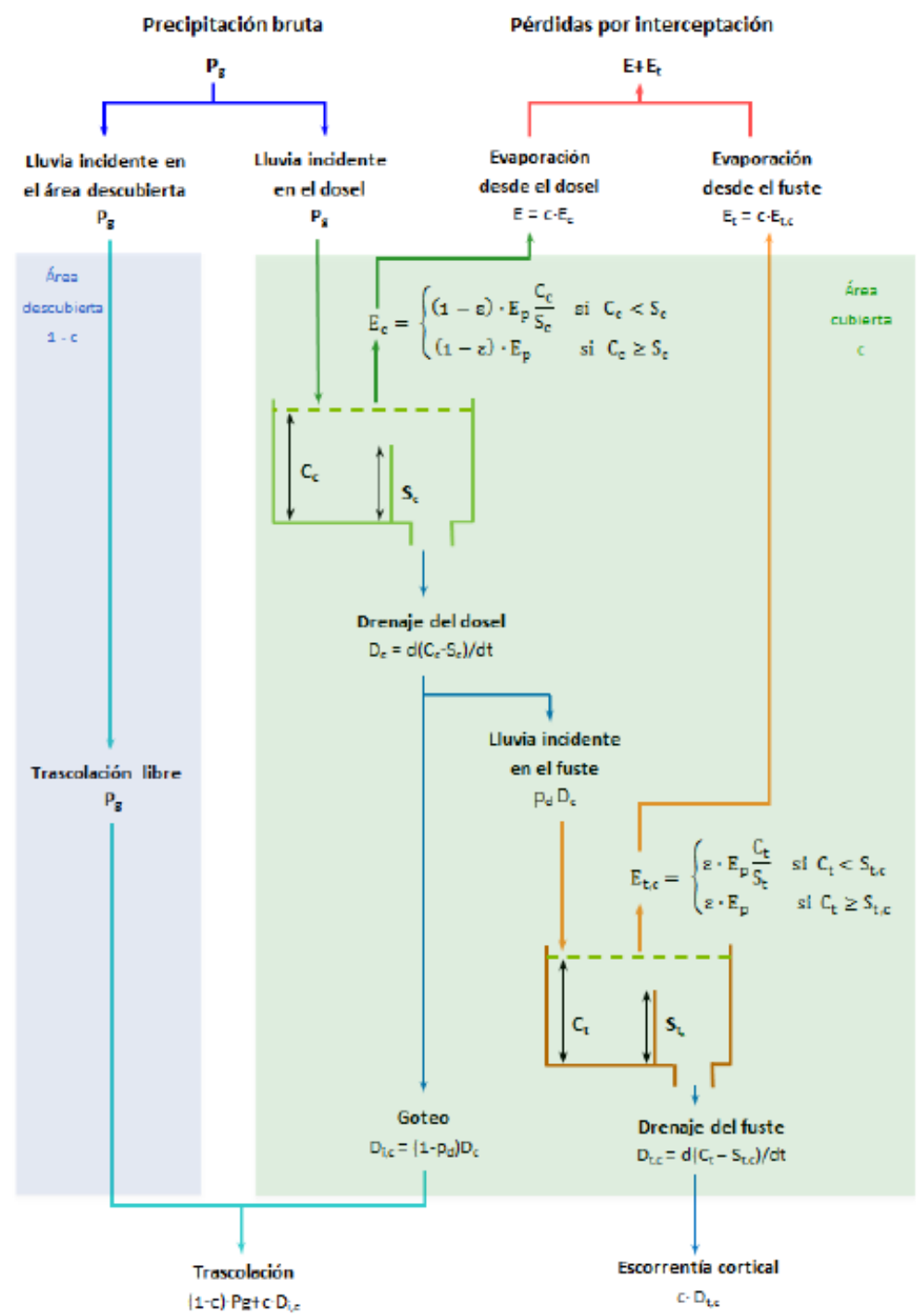

Figura 2. Modelo de Rutter modificado por Valente et al. (1997)

Tabla 2.- Componentes de la interceptación en el modelo de Gash revisado por Valente 


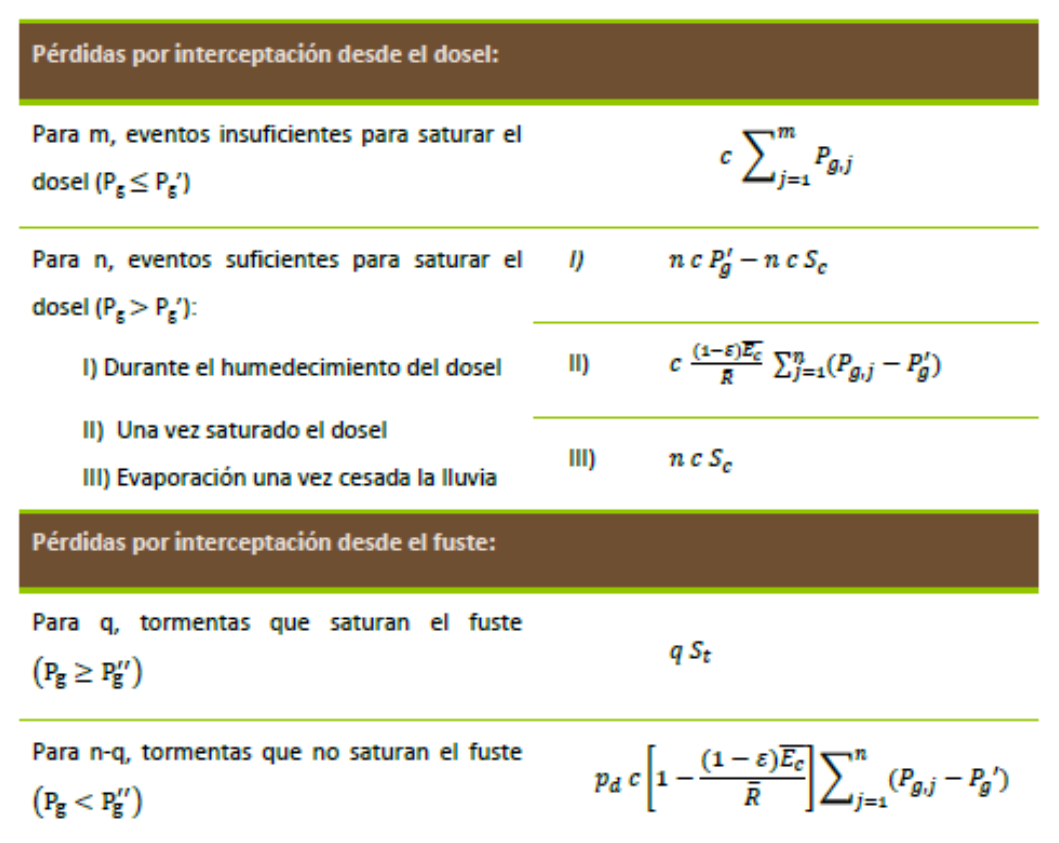

El primer paso para aplicar los modelos de interceptación fue determinar los coeficientes que participan en ellos: la capacidad de almacenamiento del dosel $(S)$ y del tronco $(S t)$, el coeficiente de trascolación libre $(p)$ y la proporción de lluvia que se desvía hacia el tronco $\left(p_{\mathrm{t}}\right)$. Estos han sido determinados por métodos gráficos.

Los coeficientes de trascolación libre $(p)$ y la capacidad de almacenamiento del dosel (S) se determinan siguiendo los criterios de Gash y Morton (1978), a partir de la representación gráfica de la precipitación bruta frente a la trascolación correspondiente a eventos individuales precedidos por $8 \mathrm{~h}$ sin lluvia (tiempo necesario para que se seque completamente el dosel), siendo $S$ el valor de la trascolación cuando la precipitación bruta es nula para eventos que saturan el dosel, mientras $p$ viene dado por la pendiente de la recta que se ajusta a los puntos representados para los eventos de lluvia insuficientes para saturar el dosel.

Los parámetros de escorrentía cortical utilizados en las versiones originales de los modelos, $S_{t}$ y $\mathrm{p}_{t}$, se obtienen siguiendo la metodología de Leyton (1967), como el punto de corte con el eje de ordenadas y la pendiente, respectivamente, de la línea de regresión entre la escorrentía cortical y la precipitación incidente. Los parámetros de escorrentía cortical para las versiones dispersas de los modelos $\left(S_{t}\right.$ y $\left.p_{d}\right)$ se estimaron a partir de la regresión lineal entre la escorrentía cortical y $\left(T_{f}-(1-c) P_{g}\right)$, de acuerdo con el método descrito por Valente et al. (1997).

\section{3- Resultados y Discusión}

En la Tabla 3 se muestran los valores de los parámetros del modelo estimados de acuerdo con los procedimientos descritos anteriormente, para las dos parcelas experimentales. Se observa una mayor capacidad de almacenamiento del dosel y una menor capacidad de almacenamiento en el tronco de Pinus pinea con respecto a Cistus ladanifer, estas diferencias se debe a la distinta morfología y arquitectura de cada especie 
Tabla 3.- Valores estimados de los parámetros para las especies Pinus pinea y Cistus ladanifer

\begin{tabular}{|c|c|c|}
\hline Parámetro & Pinus pinea & Cistus ladanifer \\
\hline$p$ & 0.304 & 0.622 \\
\hline$S(\mathrm{~mm})$ & 3.435 & 0.899 \\
\hline$p_{\mathrm{t}}$ & 0.004 & 0.184 \\
\hline $\mathrm{S}_{\mathrm{t}}(\mathrm{mm})$ & 0.013 & 0.095 \\
\hline $\mathrm{p}_{\mathrm{d}}$ & 0.012 & 0.504 \\
\hline$\varepsilon$ & 0.02 & 0.02 \\
\hline
\end{tabular}

Los valores totales de la precipitación bruta, trascolación, escorrentía cortical y la pérdida de la interceptación de las parcelas de pinos y eucaliptos, desde Octubre de 2010 hasta el Junio de 2014 se muestran en la tabla 4. La pérdida de interceptación se obtuvo como la diferencia entre la precipitación y la suma de la trascolación más la escorrentía cortical. La pérdida total intercepción fue mayor en la parcela del pino $(29.6 \%$ de la precipitación incidente) que en la parcela de la jara (17\% de la precipitación incidente).

Tabla 4.- Valores de las medidas acumuladas de precipitación incidente, trascolación, escorrentía cortical e interceptación para el período de Octubre de 2010 a Junio de 2014.

\begin{tabular}{|c|c|c|c|c|c|c|c|}
\hline \multirow{2}{*}{$\begin{array}{c}\text { Parcela } \\
\text { experimental }\end{array}$} & \multirow{2}{*}{$\begin{array}{c}\text { Precipitación } \\
\text { incidente }(\mathrm{mm})\end{array}$} & \multicolumn{2}{|c|}{ Trascolación } & \multicolumn{2}{c|}{$\begin{array}{c}\text { Escorrentía } \\
\text { cortical }\end{array}$} & \multicolumn{2}{|c|}{ Interceptación } \\
\cline { 3 - 8 } & & $\mathrm{mm}$ & $\% \mathrm{P}$ & $\mathrm{mm}$ & $\% \mathrm{P}$ & $\mathrm{mm}$ & $\% \mathrm{P}$ \\
\hline Pinus pinea & 2083.6 & 1460.0 & 70.1 & 7.0 & 0.3 & 616.6 & 29.6 \\
\hline Cistus ladanifer & 1334.2 & 877.5 & 65.8 & 229.3 & 17.2 & 227.4 & 17.0 \\
\hline
\end{tabular}

Los resultados obtenidos con las versiones originales y para bosques dispersos tanto del modelo de Rutter y del modelo de Gash se muestran en la Tabla 5. Se puede observar que los resultados obtenidos por los modelos se ajustan muy bien a los datos obtenidos en campo, especialmente en el caso de Pinus pinea, donde ninguno de los resultados de los modelos difieren más del $6 \%$ respecto las medidas observadas en campo.

Tabla 5.- Resultados obtenidos de la aplicación de los diferentes modelos en el presente trabajo (los valores entre paréntesis son la diferencia en porcentaje respecto del valor observado)

\begin{tabular}{|c|c|c|c|c|}
\hline \multirow{2}{*}{ Modelo } & \multicolumn{2}{|c|}{ Interceptación simulada $(\mathrm{mm})$} & \multicolumn{2}{c|}{ Interceptación simulada (\%) } \\
\cline { 2 - 5 } & Pinus pinea & Cistus ladanifer & Pinus pinea & Cistus ladanifer \\
\hline Rutter (1975) & $642.9(+1.3 \%)$ & $319.2(+6.9 \%)$ & 30.9 & 23.9 \\
\hline Gash (1979) & $649.4(+1.6 \%)$ & $365.2(+10.3 \%)$ & 31.2 & 27.4 \\
\hline Rutter mod. Valente (1997) & $572.2(-1.2 \%)$ & $202.4(-1.9 \%)$ & 28.7 & 25.2 \\
\hline Gash mod. Valente (1997) & $508.4(-5.2 \%)$ & $172.1(-4.1 \%)$ & 24.4 & 12.9 \\
\hline
\end{tabular}

Tal como se observa en la figura 3, cuando se reformulan los dos modelos para explicar la naturaleza dispersa de los bosques, disminuye la interceptación simulada. El modelo de Gash es el que realiza peores predicciones sobreestimando en gran medida las pérdidas por interceptación. El modelo de Rutter revisado por Valente aunque subestima la interceptación que tiene lugar en ambas especies, es el modelo que mejor se ajusta a las observaciones realizadas en campo, corrigiendo la sobreestimación del modelo original. 


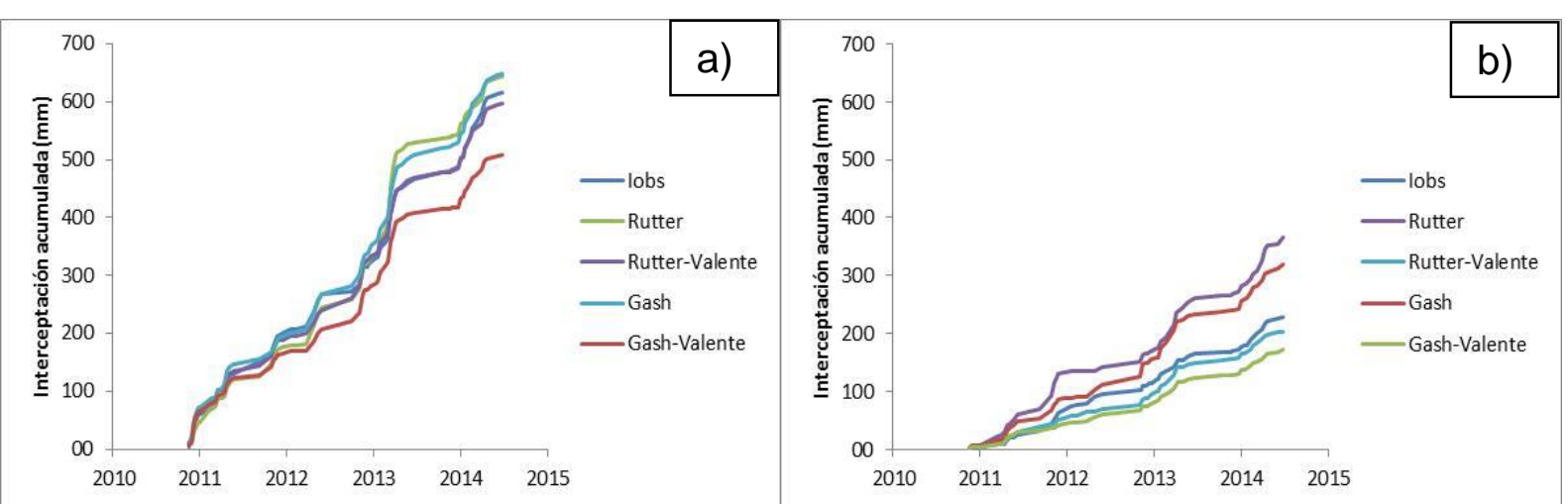

Figura 3. Interceptación medida acumulada en comparación con la estimada para las versiones originales y dispersas en Pinus pinea (a) y Cistus ladanifer(b), durante 2010-2014.

\section{4- Conclusiones}

Todos los parámetros meteorológicos y de vegetación derivados para el modelo de análisis de la pérdida de interceptación se estimaron por separado para cada especie. Se observa una mayor capacidad de almacenamiento del dosel y una menor capacidad de almacenamiento del fuste en Pinus pinea en comparación con Cistus ladanifer, valores acordes a las características de cada especie.

Los resultados de la modelización muestran claramente que las versiones originales de los modelos tanto de Rutter como Gash sobreestiman significativamente la pérdida de interceptación en las dos parcelas estudiadas, ya que estas versiones de los modelos no tienen en cuenta que la zona de evaporación real (dosel + troncos) es más reducida en los bosques dispersos.

Tanto en el caso de Pinus pinea como en el de Cistus ladanifer, el modelo de Gash es el que muestra unos valores más alejados a los medidos en campo, obteniéndose unos resultados que sobreestiman las pérdidas por interceptación.

Se ha obtenido un buen ajuste entre la observación y la predicción cuando el modelo Rutter se reformula para dar cuenta de la naturaleza dispersa del bosque. Las versiones revisadas por Valente de los modelos de Rutter y Gash, en comparación con las versiones originales, mejoran la estimación de la pérdida total de interceptación.

El modelo de Rutter revisado por Valente es el modelo que mejor se ajusta a los valores de interceptación medidos en campo. Los resultados de este modelo indican una buena correlación entre los valores de interceptación modelados y los experimentales, siendo por tanto el más recomendable para el cálculo de las pérdidas de interceptación que tienen lugar en bosques dispersos en este tipo de clima.

La principal debilidad de las versiones reformuladas de ambos modelos es probablemente el supuesto de que la evaporación para el dosel saturado de un bosque disperso se puede estimar adecuadamente por la ecuación de Penman-Monteith. Sin embargo, los resultados obtenidos aquí sugieren que esto es probablemente una simplificación adecuada y aceptable. 


\section{5.- Agradecimientos}

Este trabajo se desarrolló en el en el marco del proyecto de investigación "Balance hídrico superficial en la cuenca hidrológica de El Cabril" financiado por ENRESA (Empresa Nacional de Residuos Radiactivos) y la Universidad de Córdoba. Los autores desean expresar su agradecimiento al personal del Departamento de Ingeniería de Suelos de ENRESA por su continua y eficaz colaboración.

\section{Referencias}

Calder, I.R. 1986. A stochastic model of rainfall interception. Journal of Hydrology, 89: 65-71. Gash J.H.C. y Morton A.J. 1978. An application of the Rutter model to the estimation of the interception loss from Thetford forest. Journal of Hydrology, 90: 1-9.

Gash J.H.C. 1979. An analytical model of rainfall interception by forest. Quarterly Journal of the Royal Meteorological Society, 105: 43-55.

Gerrits A.M.J. 2010: The role of interception in the hydrological cycle. Tesis doctoral. Delft University of Technology, Holanda.

Horton R. 1919. Rainfall interception. Monthly Weather Review, 47: 603-623.

Leyton L., Reynolds E.R.C. y Thompson F.B. 1967. Rainfall interception in forest and moorland. In: W.E. Sopper and H.W. Lull (Editors), International Symposium on Forest Hydrology. Pergamon, Oxford, pp. 163-178.

Lloyd C.R., Gash J.H.C., Shuttleworth W.J. y Marques A.O. 1988. The measurement and modelling of rainfall interception by Amazonian rainforest. Agricultural and Forest Meteorology, 43: 277-294.

Monteith J.L. 1965. Evaporation and the environment. In Symposium of the Society of Experimental Biology, 19: 245-269.

Moreno-Pérez M.F., Serrano-Gómez A. y Roldán J. 2014. Application of interception models in a watershed with Mediterranean type climate. EGU General Assembly. Viena (Austria).

Mulder J.P.M. 1985. Simulating interception loss using standard meteorological data. In: B.A. Hutchison and B.B. Hicks (Editors). The Forest-Atmosphere Interaction. D. Reidel, Dordrecht, Netherlands, pp. 177-196.

Murakami S. 2007. Application of three canopy interception models to a young stand of Japanese cypress and interpretation in terms of interception mechanism. Journal of Hydrology. 342: 305-319.

Muzylo A., Llorens P. y Domingo F. 2012. Rainfall Partitioning in a Deciduous Forest Plot in Leafed and Leafless Periods. Ecohydrol., 5: 759-767.

Pereira F.L., Gash J.H.C., David J.S., David T.S., Monteiro P.R. y Valente F. 2009. Modelling interception loss from evergreen oak Mediterranean savannas: Application of a treebased modeling approach. Agricultural and Forest Meteorology. 149: 680-688. 
Rutter A.J. Kershaw K.A., Robins P.C. y Morton A.J. 1971. A predictive model of rainfall interception in forest. I. Derivation of the model from observations in a plantation of Corsican pine. Agricultural Meteorology, 9: 367-384.

Rutter A.J., Morton A.J. y Robins P.C. 1975. A predictive model of rainfall interception in forest. II. Generalization of the model and comparison with observations in some coniferous and hardwood stands. Journal of Applied Ecology, 12: 367-380.

Serrano A. 2013. Uso de moledos de interceptación de lluvia en la cuenca hidrológica de "El Cabril" (Córdoba). Trabajo Profesional Fin de Carrera. Universidad de Córdoba.

Valente F.; David J. y Gash J. 1997. Modelling interception loss for two sparse eucalypt and pine forests in central Portugal using reformulated Rutter and Gash analytical models. Journal of Hydrology, 190: 141-162.

Wallace J. y McJannet D. 2006. On interception modeling of a lowland coastal rainforest in northern Queensland, Australia. Journal of Hydrology. 329: 477-488.

Whitehead D. y Kelliher F.M. 1991. A canopy water balance model for a Pinus radiata stand before and after thinning. Agile. For. Meteorol., 55: 109-126.

Zhang G., Zeng G.M., Jiang Y.M., Huang G.H., Li J.B., Yao J.M., Tan W., Xiang R.J. y Zhang X.L. 2006. Modelling and measurement of two-layer canopy interception losses in a subtropical mixed forest of central-south China. Hydrology and Earth System Sciences 10: 65-77. 